\title{
Multisystem Inflammatory Syndrome in Children Presenting as Subacute Intestinal Obstruction
}

\author{
Sunil Joghee ${ }^{1}$ (1) $\cdot$ Neminathan Kanthasamy ${ }^{1} \cdot$ Senthil Kumar Lakshmanan ${ }^{1}$
}

Received: 30 July 2021 / Accepted: 20 August 2021 / Published online: 6 September 2021

(c) Dr. K C Chaudhuri Foundation 2021

To the Editor: A 9-y-old girl was admitted with fever and vomiting for $3 \mathrm{~d}$, not passing stools for $2 \mathrm{~d}$, abdominal pain and fullness for $1 \mathrm{~d}$. She was febrile $\left(105^{\circ} \mathrm{F}\right)$, dehydrated, and tachycardic; there was abdominal distension with generalized tenderness and sluggish bowel sounds. Abdominal radiograph showed multiple air fluid levels in the abdomen suggestive of intestinal obstruction. She was kept nil by mouth with continuous nasogastric tube aspiration which showed minimal bilious aspirates. She was started on intravenous fluids and empirical antibiotics pending cultures.

Blood investigations showed neutrophilic leukocytosis, mild thrombocytosis, and elevated erythrocyte sedimentation rate (ESR - $60 \mathrm{~mm} / \mathrm{h}$ ) and C-reactive protein (CRP $31 \mathrm{mg} / \mathrm{dL}$ ); urinalysis was normal. Serum lipase and amylase were mildly raised and transaminases were normal. RT-PCR for severe acute respiratory syndrome coronavirus 2 (SARS-CoV-2) was negative. Serology for SARS-CoV-2 showed elevated IgG titer (538 AU/mL). Further investigation revealed elevated troponin-T, N-terminal prohormone of brain natriuretic peptide (NT pro-BNP) and D-dimer. Echocardiography showed mild left ventricular dysfunction. Dengue serology and Typhidot IgM were negative. Blood culture was sterile. A diagnosis of multisystem inflammatory syndrome in children (MIS-C) was made as she fulfilled the WHO criteria [1]. She was treated with intravenous immunoglobulin (IVIG), low-dose methyl-prednisolone and aspirin. Her symptoms resolved gradually. Repeat inflammatory markers and echocardiography were normal at $2 \mathrm{wk}$ follow-up.

Gastrointestinal (GI) symptoms like vomiting, diarrhea, and abdominal pain have been reported in up to $90 \%$ children with MIS-C [2]. However, there are only a few case reports of MIS-C presenting with features of intestinal

Sunil Joghee

sunilkapv@gmail.com

1 Department of Pediatrics, Coimbatore Child Trust Hospital, Singanallur, Coimbatore, Tamil Nadu 641005, India obstruction $[3,4]$. To the best of our knowledge, this is the first case of MIS-C presenting with features of intestinal obstruction reported in India. We believe MIS-C should be considered in the differential diagnosis of children presenting with features of intestinal obstruction, especially during the ongoing pandemic.

\section{Declarations}

Consent for Publication A written informed consent was obtained from the parents to share clinical information about the patient.

Conflict of Interest None.

\section{References}

1. WHO. Multi system inflammatory syndrome in children and adolescents temporally related to COVID-19. Available at: https://www.who.int/ news-room/commentaries/detail/multisystem-inflammatory-syndromein-children-and-adolescents-with-covid-19. Accessed on 5 July 2021.

2. Feldstein LR, Tenforde MW, Friedman KG, et al. Characteristics and outcomes of US children and adolescents with multisystem inflammatory syndrome in children (MIS-C) compared with severe acute COVID-19. JAMA. 2021;325:1074-87.

3. Sahn B, Eze OP, Edelman MC, et al. Features of intestinal disease associated with COVID-related multisystem inflammatory syndrome in children. J Pediatr Gastroenterol Nutr. 2021;72:384-7.

4. Morparia K, Park MJ, Kalyanaraman M, McQueen D, Bergel M, Phatak T. Abdominal Imaging findings in critically Ill children with multisystem inflammatory syndrome associated with COVID-19. Pediatr Infect Dis J. 2021;40:E82-3.

Publisher's Note Springer Nature remains neutral with regard to jurisdictional claims in published maps and institutional affiliations. 